\title{
Breast reconstruction using internal mammary artery perforator (IMAP) flap
}

\author{
Kareem Abdelmonem, ${ }^{a} M D ;$ MRCS, Ahmed Elshahat, ${ }^{a} M D$; \\ Hossam Abol-Atta, ${ }^{a} M D$; Sherif Abou-Gamrah, ${ }^{b} M D$; \\ Reda Abd Eltawab, ${ }^{c} M D$; Karim Massoud, ${ }^{a} M D$
}

a) Department of Plastic Surgery, Ain Shams University, Cairo, Egypt.

b) Department of Radiodiagnosis, Ain Shams University, Cairo, Egypt.

c) Department of General Surgery, Ain Shams University, Cairo, Egypt.

\section{Corresponding Author:}

Hossam Abol-Atta, M.D.

Plastic Surgery Department, Faculty of Medicine, Ain Shams University, Abbassia, 11566, Cairo, Egypt

Email:hosaatta@yahoo.com

\begin{abstract}
Post mastectomy reconstruction using the internal mammary artery perforator (IMAP) flap, previously called breast-sharing technique, is an appealing procedure because it utilizes the tissue to be excised during reduction of the residual breast to achieve symmetry. Literature mentioned that this tissue is supplied by the lower IMAPs with no further details. The aim of this study was to re-explore the old theme of breast-sharing by describing the IMAPs included in the pedicle of this breast-derived flap via using color Duplex ultrasound preoperatively, thus re-introducing the flap under a name relating to its exact vascular supply, that is, IMAP flap. Six breasts of six patients presented for post mastectomy reconstruction were preoperatively screened via color duplex, and were operated on. Three cases (50\%) underwent completely successful IMAP flap breast reconstruction, two (66.7\%) experienced partial flap loss, and one patient (16.7\%) had total flap necrosis. We see the IMAP flap deserves to be within the repertoire of the plastic surgeon to be prudently employed in the properly selected patients.

Key words: Internal mammary artery perforator flap; IMAP; breast reconstruction; color Duplex; breast sharing; cross-breast flap.
\end{abstract}

\section{Introduction:}

The emotions surrounding a breast cancer diagnosis are complex, but patients generally have two dominant concerns, preservation of life and preservation of body form. The plastic surgeon addresses the latter concern by restoring the breast form through choosing between multiple surgical procedures including either the use of a mammary prosthesis (implant) or various autologous tissues.

Regarding autologous reconstruction, the lowest donor site morbidity could be achieved by using tissue that would be otherwise discarded during an operation necessary for a different reason. Adoption of this principle was the cause behind the introduction of the transverse rectus abdominis musculocutaneous (TRAM) flap for breast reconstruction. ${ }^{1,2}$ Similarly, in post mastectomy patients in whom the remaining breast has to be reduced anyway because of hypertrophy, such surplus breast tissue has been reported to be used as a pedicled flap 
for the missing breast reconstruction. . $^{3-7}$

Many studies agreed on the dominant role of the internal mammary artery (IMA) in nourishing the ipsilateral ventromedial twothirds of the chest wall and breast through its perforating branches, namely internal mammary artery perforators (IMAPs). ${ }^{8-12}$ On the ground of such information, a number of works introduced a set of IMAPbased cutaneous flaps (glandulo-cutaneous in women) with variable reconstructive applications for the breast, ${ }^{3-7,13-15}$ the anterior chest wall, and head and neck regions. ${ }^{16-21}$ Some works $9,11,20,22,23$ stated that the $4^{\text {th }}$ IMAPs are the main perfusing vessels to the lower part of the breast while the 5th ones are responsible for supplying the tissues caudal to the inframammary folds. The articles relating to the lower IMAPs-based flaps used for the chest wall and the contralateral breast reconstruction ${ }^{3-7,13-17}$ presented no precise data about the IMAPs included in these flap pedicles.

Doppler ultrasonography has been proven to be a highly valuable and practical tool for preoperative mapping of perforating vessels throughout the cutaneous territory of a flap, aiming at improving the surgical strategies so that the operative procedures can proceed in a safer way. ${ }^{24-26}$ Schoeller et al. ${ }^{7}$ and Dian et al. ${ }^{15}$ conducted doppler sonographic studies for the internal mammary artery perforators before elevating flaps based on them. They detected the perforators but gave no details regarding their number, size or exact location. In a recent clinical study (not anatomical) performed by us, ${ }^{23}$ we conducted preoperative color Duplex screening for the lower IMAPs, namely the $4^{\text {th }}$ and $5^{\text {th }}$ ones, supplying the inferior breast tissue typically excised in reduction mammoplasty cases and presented a detailed description relating to the location, number and diameters of these perforators.

The aim of current study was to attempt at reviving as well as reconceptualizing the old theme of employing the intact breast as a donor site for reconstructing the contralateral ablated one, with a preoperatively-identified IMAP-pedicle of the breast tissue used.

\section{Patients and methods:}

This study was conducted on 6 female patients who presented for unilateral post mastectomy reconstruction and still had intact contralateral breast. A signed written informed consent was obtained from each patient regarding her agreement on participation in this study, and explaining that in the event of intra- or postoperative flap failure, a latissimus dorsi musculocuatneous flap reconstruction would be performed, and if postoperative oncological follow-up detected a new cancer formation either in the donor or in the reconstructed breast, it can be removed and reconstructed again using the other standard, available (still-not-depleted) options.

Preoperatively, the $4^{\text {th }}$ and $5^{\text {th }}$ intercostal spaces on the residual breast side only were screened parasternally for perforating vessels by color Duplex. That was to ensure the presence of at least one IMAP in the $4^{\text {th }}$ interspace perfusing the inferior tissue of the planned reduction mammoplasty on this intact breast, and subsequently, the possible employment of such tissue for contralateral side reconstruction through a multi-staged procedure.

\section{Sonographic technique:}

A color Duplex scanner (LOGIQ 7 PRO: General Electric Yokogawa Medical Systems Ltd., Tokyo, Japan) was used to preoperatively visualize the site (distance from the lateral sternal border), the diameter, and identify the number of detectable IMAPs (if any) in each of the above two mentioned intercostal spaces. To avoid potential errors caused by different interpretations, Duplex was done by a single radiologist (AbouGamrah), experienced in Duplex assessment of small vessels.

At first, each patient was positioned in the supine position, and then the intercostal spaces from the 2nd-5th were marked on the residual breast side only Figure(1). Parasternal regions at the $4^{\text {th }}$ and $5^{\text {th }}$ spaces were scanned using B-mode ultrasonography with a linear probe frequency of $12 \mathrm{MHz}$ after adjusting the B-mode gain to clearly 
visualize the deep fascia, then a color Duplex with pulsed Doppler wave was used to detect the perforators after adjusting the following parameters: pulse repetition frequency at low setting level to detect low velocities; color gain to avoid over or under estimation of the perforator's diameter; the Doppler angle to be less than 60 degrees; and the sample volume of the Doppler beam. The remoteness of the perforator site from the sternum, and the number of encountered perforators parasternally in each investigated space were identified. The inner diameter of each perforator was measured at its emergence point from the surface of the overlying pectoralis muscle Figure(2). Data were recorded for analysis.

\section{Surgical technique:}

A superior pedicle reduction mammoplasty with inverted-T pattern was performed to the residual breast of all the participants. As proceeding from one patient to another, the used technique was subjected to ameliorative modifications aiming at gaining better results and avoiding the complications encountered with the earlier cases. The most important amendment applied was adding a preliminary surgical delay stage to the procedure, hence turning the maneuver to be three- rather than two-staged. The survived flap surface area [length $(\mathrm{cm}) \mathrm{x}$ width $(\mathrm{cm})$ ] was recorded for analysis.

For the early 2 consecutive cases, reconstruction was performed through two stages; flap transposition followed 6 weeks later by flap pedicle division and donor breast closure. Firstly, after marking the donor breast for the reductive surgery Figure(3), the inferior lipoglandulo-cutaneous tissue, that was planned to be discarded in such reduction mammoplasty, was elevated off the underlying muscle as a flap, starting the dissection from the axillary side and proceeding medially till about $3 \mathrm{~cm}$ before the ipsilateral sternal border to avoid injuring the supplying IMAPs, and then rotated about $180^{\circ}$ across the midline, while still attached to the ipsilateral parasternal area Figure(4). Simultaneously at this stage, the planned NAC-pedicle on the donor breast was de-epithelialized, dissected and then transferred up to its planned new level Figure(6E\&F).

The skin over the sternum which lay under the flap base was transversely incised to accommodate the raw back surface of the part of the flap bridging over the sternum Figure $(6 E \& F)$. This last step was also done in the $3 \mathrm{rd}$ patient in the rank of cases in addition to utilizing skin graft to share in covering this raw part of the flap. After six weeks, the second stage of flap pedicle division was performed with accomplishing closure of the reduced donor breast Figure(5).

Owing to the vascular complications encountered with the flap in the earliest 2 cases alluded to above, the modified threestage procedure was adopted in the subsequent reconstruction patients. Here, the first stage involved a delay procedure for the lateral half of the inferior mammary tissue as follows; this part was incised, dissected and raised all around except medially, and then sutured in place in order to enhance its vascularity. Two weeks later, a second stage of completing this inferior tissue segment elevation and acrossmidline transposition was done. After another 6 weeks, the final stage of flap pedicle division and donor breast remodeling was done.

Also, starting from the $4^{\text {th }}$ case onwards, the incision used to be done over the sternum was abandoned, and the de-epithelialization of the NAC-carrying pedicle of the donor breast was postponed as well till the last stage of the procedure, to be done with donor breast reshaping and closure. Split-thickness skin grafting was resorted to for covering the raw back surface at the flap base and thus a median tunnel has been created Figure(4). But again, this was avoided in the next 2 cases due to the poor taking of the skin graft by the fatty raw area on the flap base back surface in addition to patients' inconvenience with the idea of skin graft harvesting from their thighs. Hence this raw area of the flap under surface was left to granulate with follow-up dressing till the last stage of flap division in the last 2 cases.

In order to preserve the original markings of the planned reduction on the breast throughout the procedure till the last stage, a scoring with a scalpel was done to these 
markings during the first stage of flap delay. Thereby, after flap pedicle division, the donor breast was remodeled and closed according to the scoring-preserved original inverted-T reduction markings Figure(5).

A latissimus dorsi musculocutaneous flap reconstruction was done for two cases (the 1 st and $2^{\text {nd }}$ case in this series respectively) in order to compensate for partial IMAP flap loss in the $1^{\text {st }}$ case and total flap loss in the $2^{\text {nd }}$ one.

On their demands, NAC reconstruction was done in three patients $(50 \%)$ few months after completion of the last stage of IMAP flap reconstruction. Re-reduction of the donor breast was done as well in two out of the above three patients upon request. In these latter two cases, nipple was reconstructed via arrow flap and the skin of the re-reduction was exploited as a split-thickness skin graft for reconstructing the areola. The remaining patient didn't ask for re-reduction of her donor breast, so only the nipple was reconstructed by arrow flap while the patient expressed her convenience with the idea of areola tattooing in the future. But, this last patient asked for correction of step-off deformity between the reconstructed breast and upper chest wall Figure $(\mathbf{8 K} \& \mathbf{L})$, so autologous fat grafting (by liposuction then injection) was done to correct this contour deformity.

\section{Data management and analysis:}

The collected data were introduced to a personal computer using statistical package for social science (SPSS 17.0.1 for windows; SPSS Inc, Chicago, IL, 2008). A p-value $<0.05$ was assumed as significant.

\section{Results:}

A $4^{\text {th }}$ IMAP-based flap was used in six post mastectomy patients aged between 30 and 45 years (mean $35.5 \pm 6.2$ years) Tables(1\&2). The flaps $(n=6)$ were transposed from the inferior half of the residual breasts to the contralateral side over multiple stages. Varying vascular complications to the flap on three occasions (50\%) were encountered. Using preoperative Duplex scanning, the $4^{\text {th }}$ IMAP was detected in all cases $(100 \%)$ while the 5 th perforator was detectable in four cases only $(66.7 \%)$ Table(1). Three patients (50 $\%$ ) had the residual IMAP-flap-donor breast on the left side and the other three had it on the right side. The NAC of the donor breasts $(\mathrm{n}=6)$ were at a mean distance of $35.8 \pm 5.4 \mathrm{~cm}$ from the suprasternal notch (ranging between 28 and $42 \mathrm{~cm}$ ) Tables(1\&2). The IMAP flap reconstruction procedure was performed over two stages in the earliest consecutive two cases $(33.3 \%)$, and then modified to become three-staged involving a preliminary surgical delay procedure to the lateral half of the flap Figure $(\mathbf{8 E \& F})$ in the remaining four patients $(66.7 \%)$.

The $4^{\text {th }}$ IMAP included in the flaps' pedicles was found to lie at a mean distance of $1.3 \pm 0.5 \mathrm{~cm}$ (range, 0.5 to $2 \mathrm{~cm}$ ) lateral to the ipsilateral sternal border, and to have a diameter ranging between 1 to $1.5 \mathrm{~mm}$ (mean, $1.2 \pm 0.2 \mathrm{~mm}) \quad$ Tables(1\&2). The average length and width of the survived 4th IMAPbased flaps $(\mathrm{n}=5$, that is, $83.3 \%)$ were $19.6 \pm 4.7 \mathrm{~cm}$ and $10.6 \pm 1.1 \mathrm{~cm}$ respectively, with average surface area of $211.4 \pm 67.7 \mathrm{~cm}^{2}$ Table(2). The longest flap survived wasn't the widest one, while the shortest flap was the narrowest one Tables(1\&2).

For the $1^{\text {st }}$ case Figure(6), partial flap necrosis occurred to the distal flap fourth, with subsequent debridement of the devitalized tissues under local anesthesia. After six weeks, during the flap pedicle division procedure and donor breast closure, a pedicled latissimus dorsi musculocutaneous flap was transposed to compensate for the IMAP flap partial loss, and the remaining medial part of the IMAP flap was de-epithelialized and embedded like a "bioprosthesis" under the transposed latissimus flap. Regarding the $2^{\text {nd }}$ case, total flap necrosis occurred. So, a latissimus dorsi musculocutaneous flap was done for the entire reconstruction. Thus, two cases $(33.3 \%)$ underwent compensatory latissimus dorsi flap reconstruction.

Despite the added stage, starting from the $3^{\text {rd }}$ case onwards, of surgical delay to the distal half of the flap Figure $(\mathbf{8 E} \& \mathbf{F})$ to overcome the aforementioned vascular insults, the $5^{\text {th }}$ case in this series experienced severe congestion to 


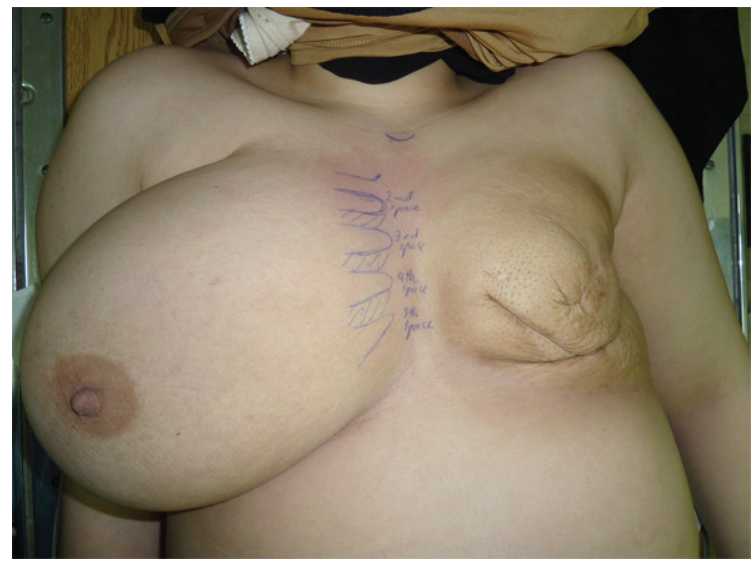

Figure (1): Anterior chest wall of a female in supine position with left-side modified radical mastectomy showing pre-Duplex marking of the 2nd through the 5th intercostal spaces on the right side of the residual breast.

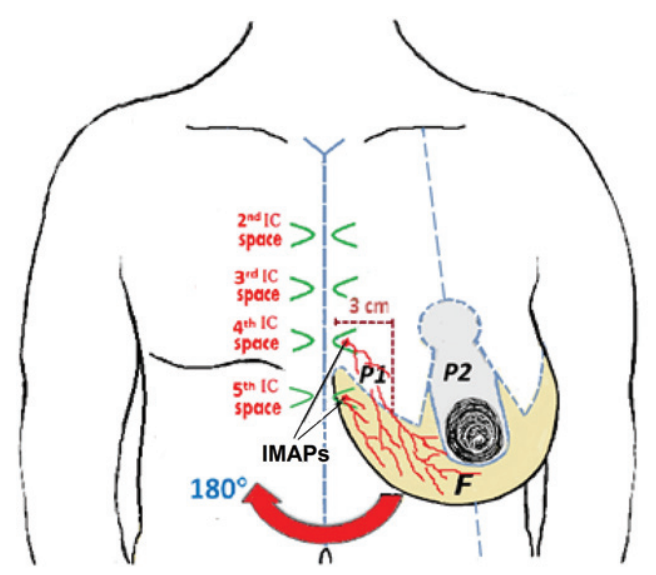

Figure (3): Drawing of the preoperative inverted-T marking of the donor breast. The "beige" colored area represents the inferior mammary tissues " $F$ " normally discarded in such reductive breast surgery, and that would be otherwise employed as an IMAP-based flap for reconstructing the contralateral side (P1: IMAP-containing pedicle of the tissue flap, P2: NACcarrying pedicle of the donor breast to be reduced, IMAPs: Internal mammary artery perforators, IC space: Intercostal space).

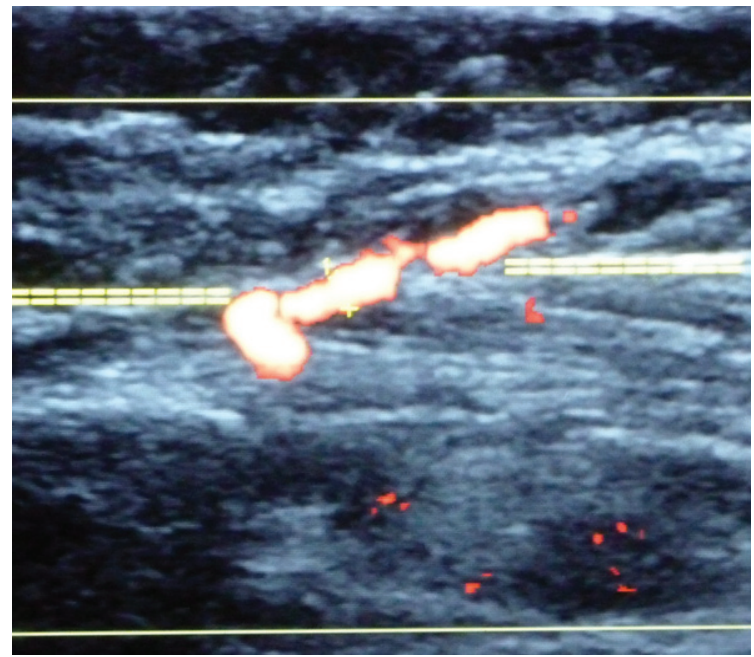

Figure (2): A Duplex photo showing an IMAP while perforating the surface of the pectoralis major muscle (the doublrdashed line). Note the two cross-shaped signs indicating the diameter to be of the perforator to be measured at its perforation point.

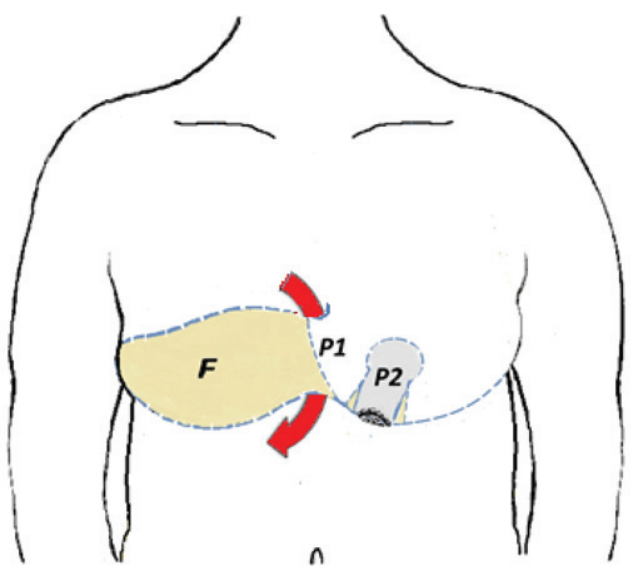

Figure (4): Drawing of the lower pole breast flap " $F$ " (beige colored) after being transposed as an IMAP-based flap to the contralateral side while still attached at the donor breast medial border "P1" which contains the flap supplying pedicle. The red arrow illustrates the median tunnel to be created below the flap base and over the sternal skin (P2: NAC-carrying pedicle of the donor breast). 


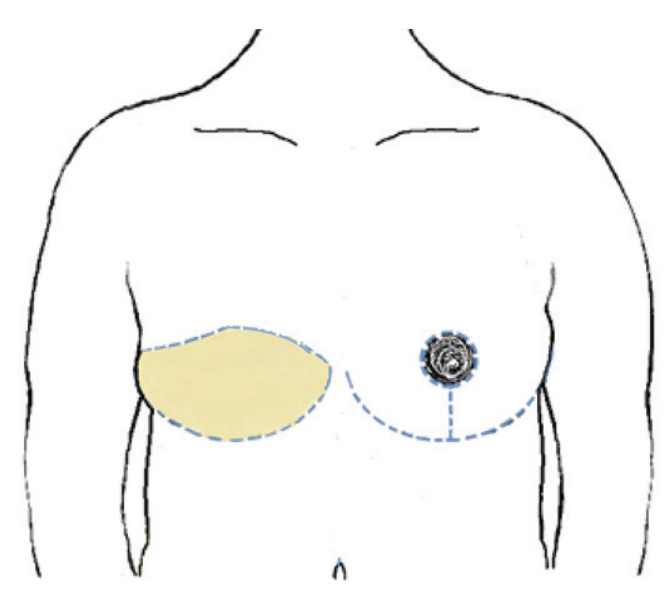

Figure (5): Drawing of the end result after the IMAP flap (beige colored) pedicle division, and closure and remodeling of the donor breast cone as with the inverted-T reduction mammoplasty technique.
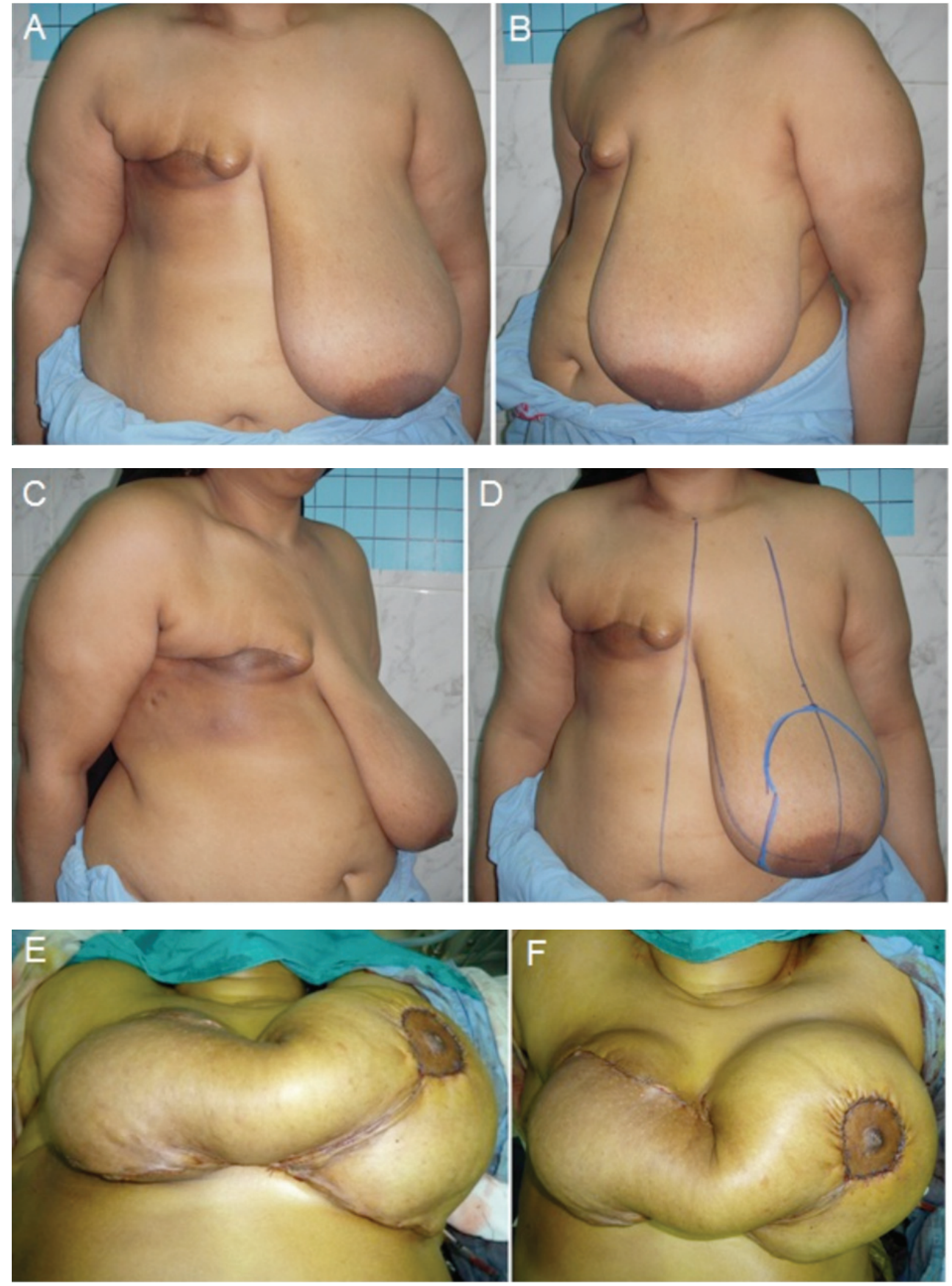

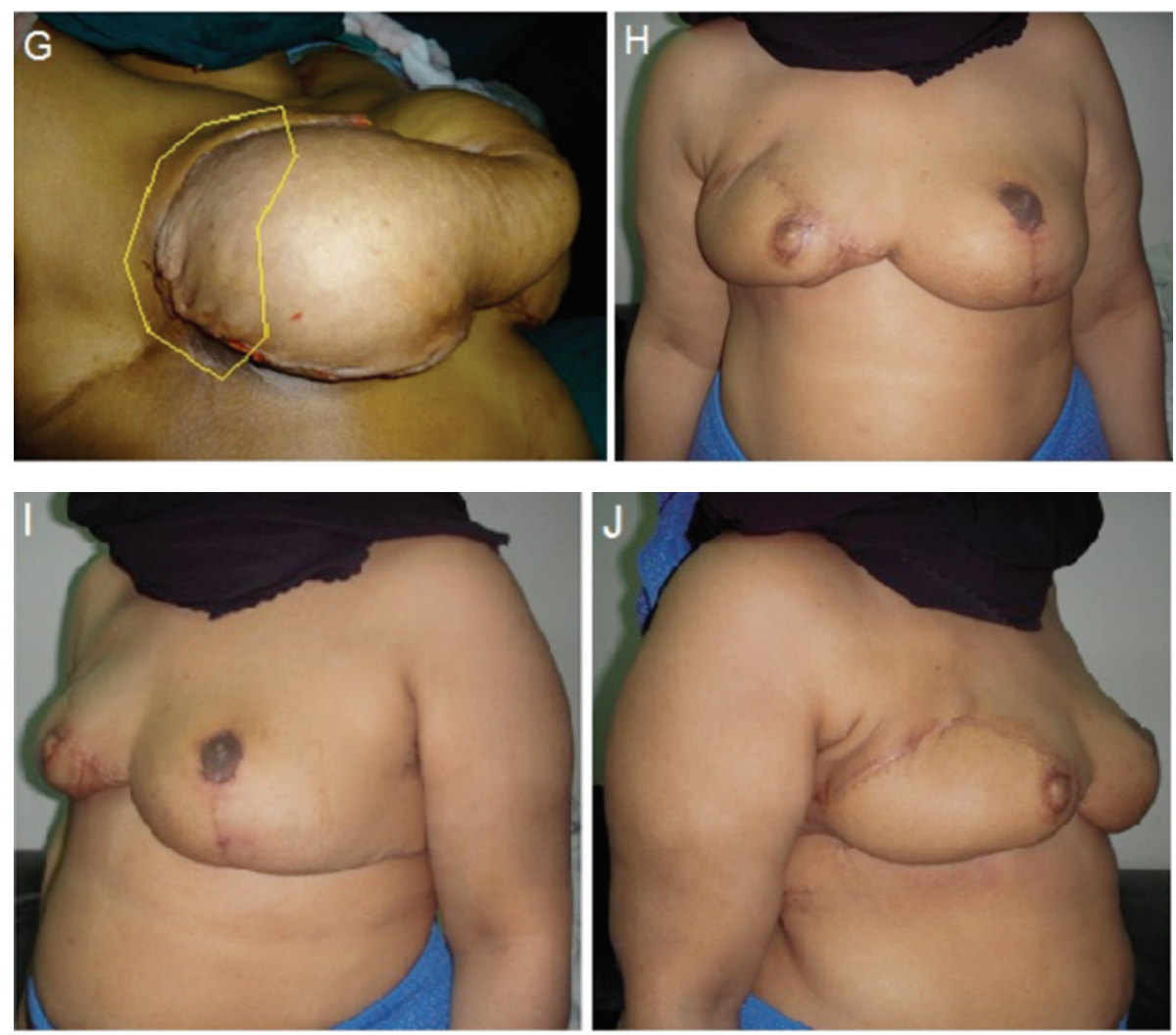

Figure (6): Pre- and intra-operative views of the 1st IMAP flap reconstruction case; ( $A, B$ $\& C)$ Front and oblique pre-operative views. (D) Preoperative marking of the donor breast. $(E \& F)$ Intra-operative views after flap transposition to the right side showing the flap part bridging over the sternum while being harbored in an area over the sternum created via a transverse incision. Also, the NAC of the donor breast was transferred to its planned position on the donor breast during this stage. (G) The distal part of the transposed flap which, later, showed ischemic changes and was debrided (the yellow-line circumscribed area). (H, I \& $J)$ Late post-operative front and oblique views (10 months) after right-side compensatory $L D F$ reconstruction. The remaining medial part of the earlier complicated IMAP flap was deepithelialized and employed as a "bioprosthesis" under the latissimus flap. Also, NAC reconstruction was done at the time of $L D F$ reconstruction by skin grafting from the rereduced left breast at that time too.
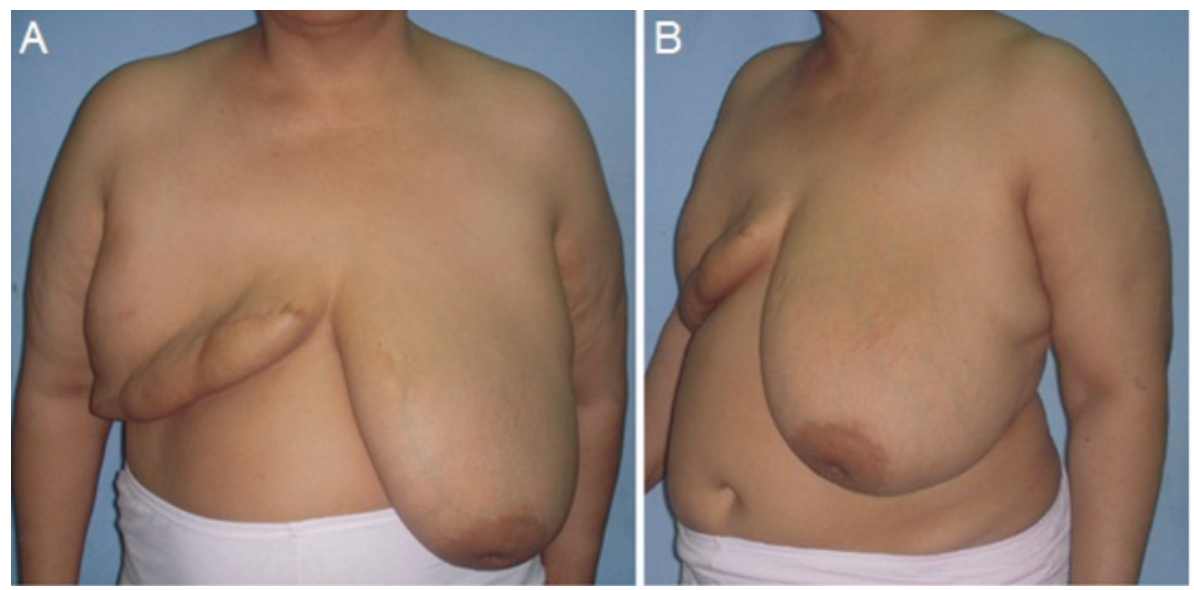

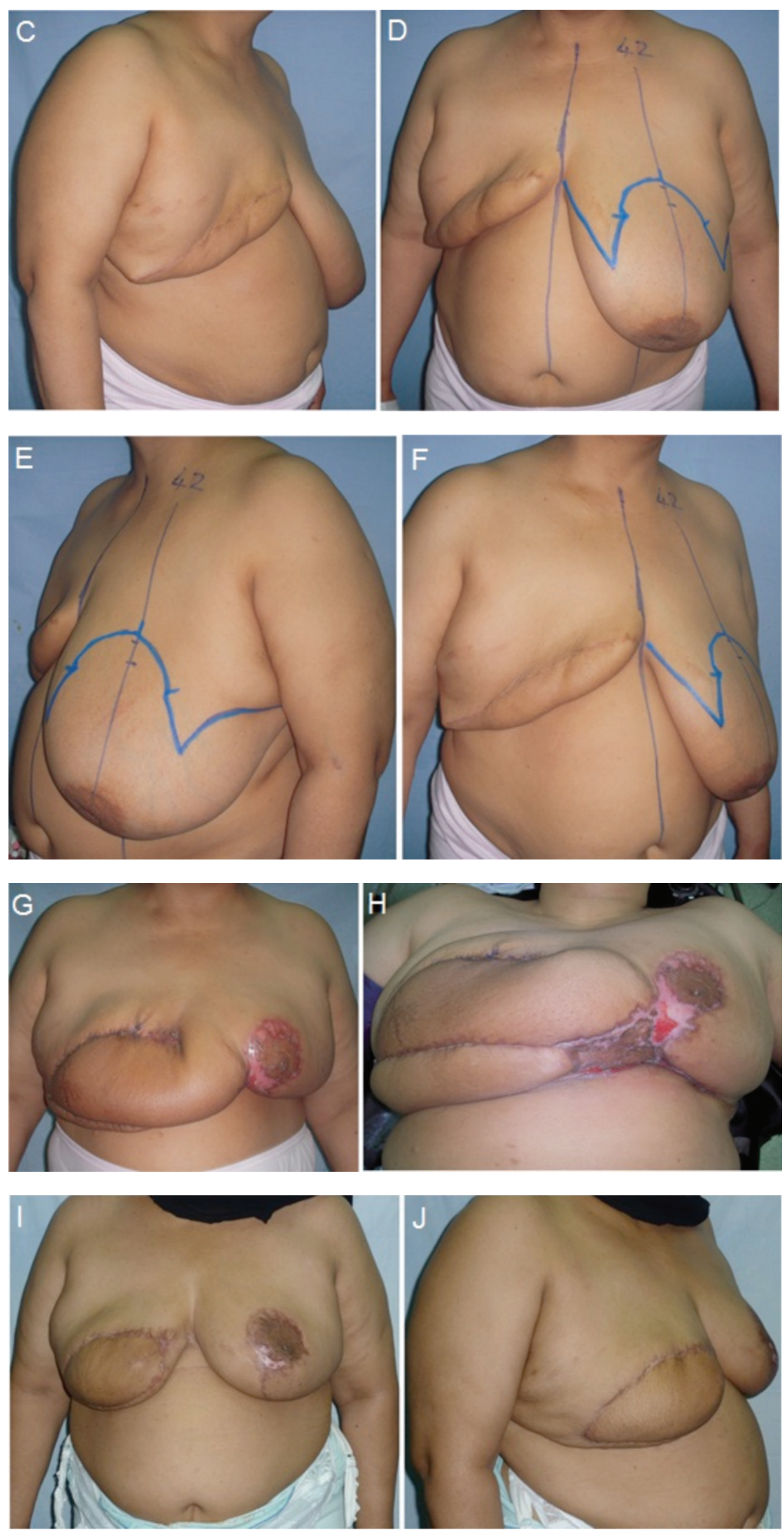


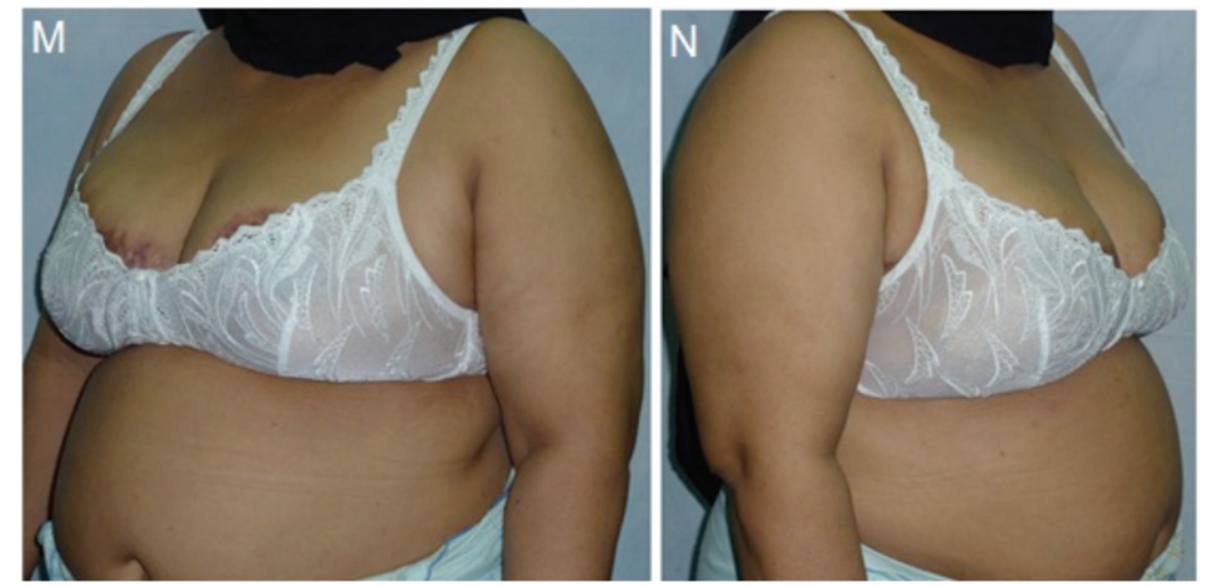

Figure (7): Pre-, intra- and post-operative views of one of the completely successful IMAP flap reconstruction patients; ( $A, B \& D)$ Front and oblique pre-operative views. (D) Preoperative markings, front view. $E \& F$ ) Pre-operative marking, oblique views. ( $G \& H)$ Pre-division views of the flap showing a split-thickness skin graft covering the raw area at the flap base. Note that the NAC was elevated to its planned site on the donor breast at the time of flap transposition with inability to completely close the pillars below it to avoid tension on the flap pedicle. This step became relegated to the final stage in the next cases. (I \& J) Post-operative views (front and oblique), without clothing, 15 weeks after completing the third stage of flap division and donor breast reshaping. (K) Another post-operative oblique view without clothing. ( $L, M \& N$ ) Post-operative views (front and oblique) showing the good contour obtained of the right reconstructed breast as well as the good symmetry achieved between both sides under clothing (worn bra).
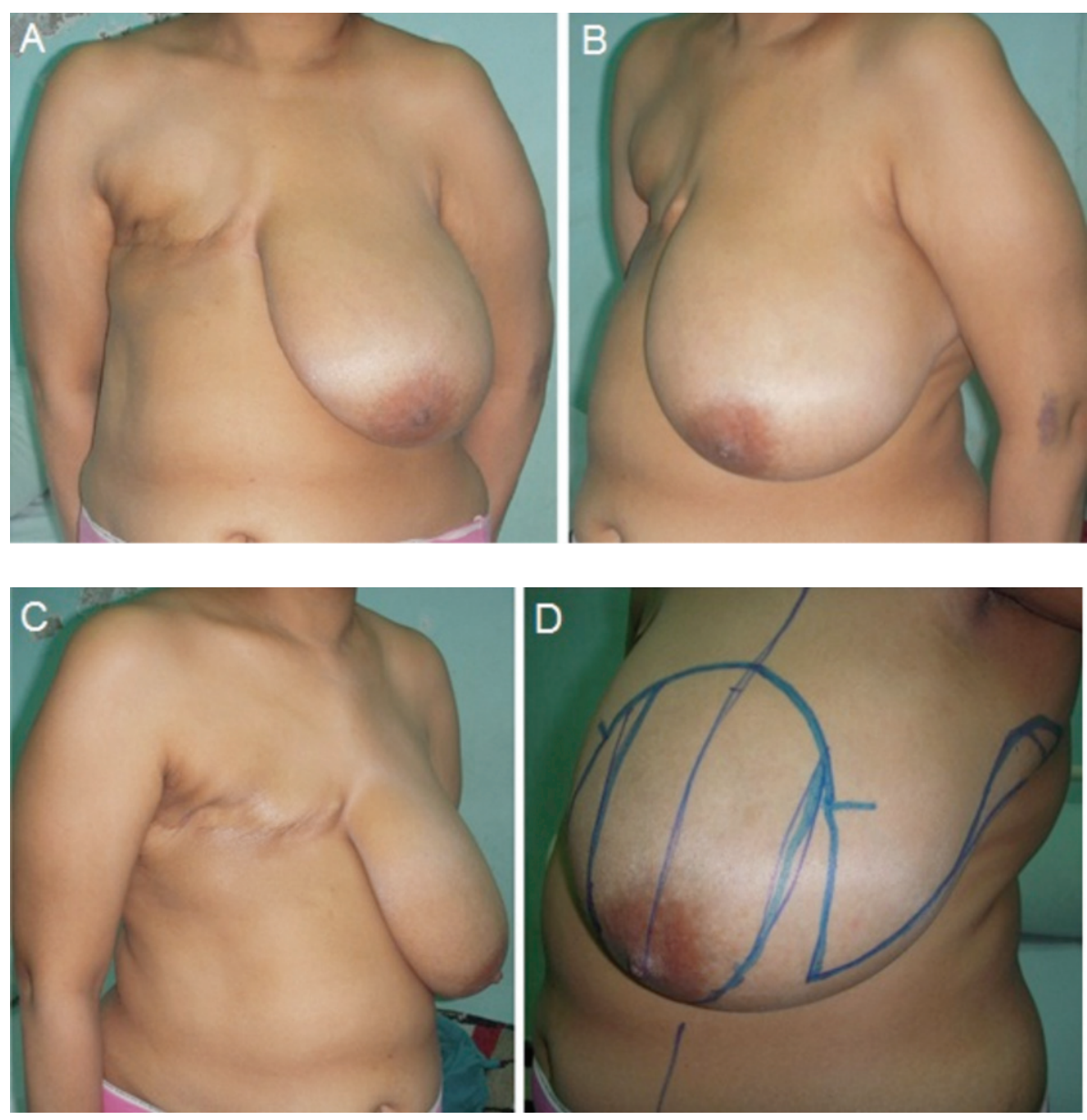

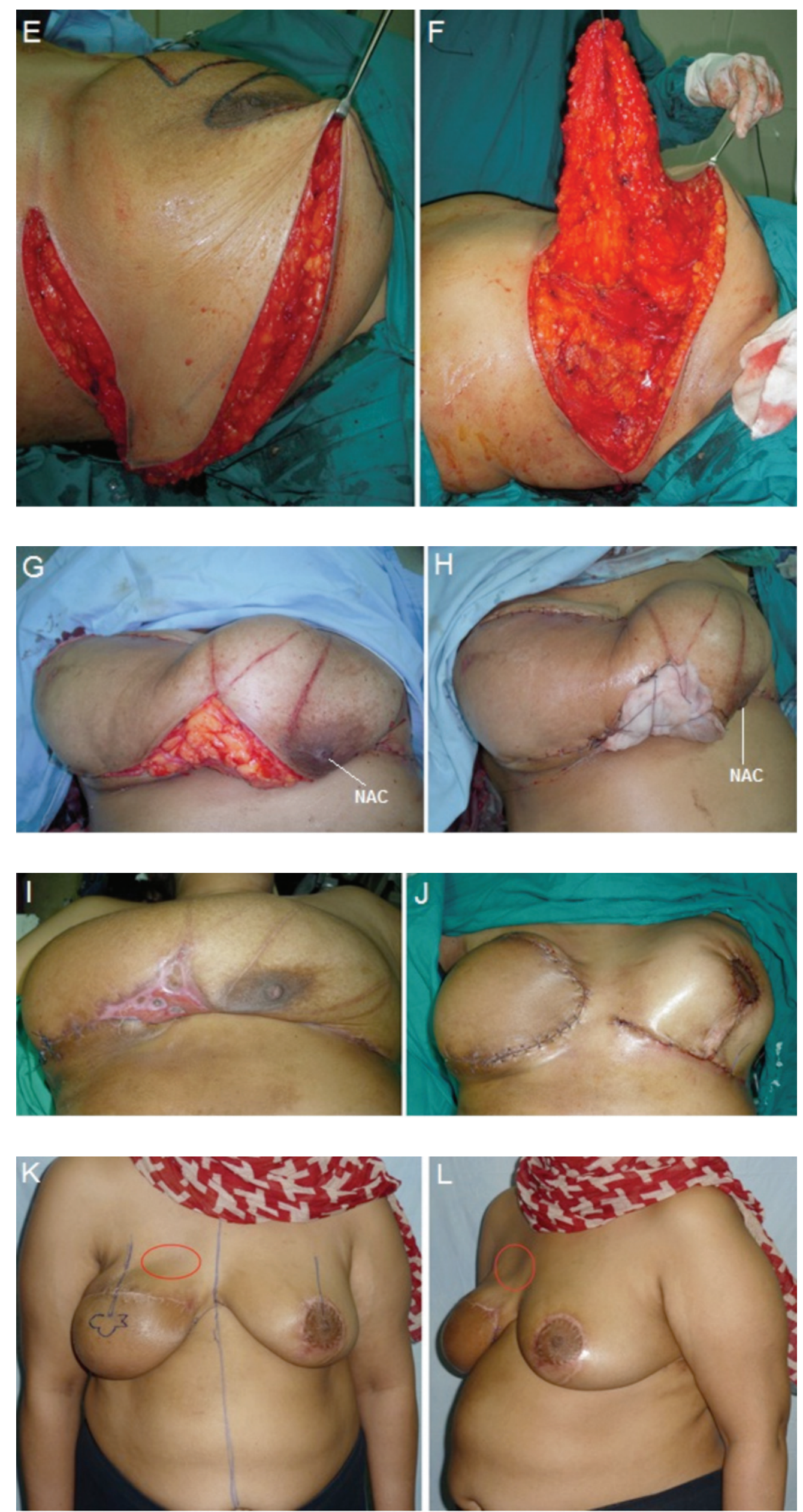

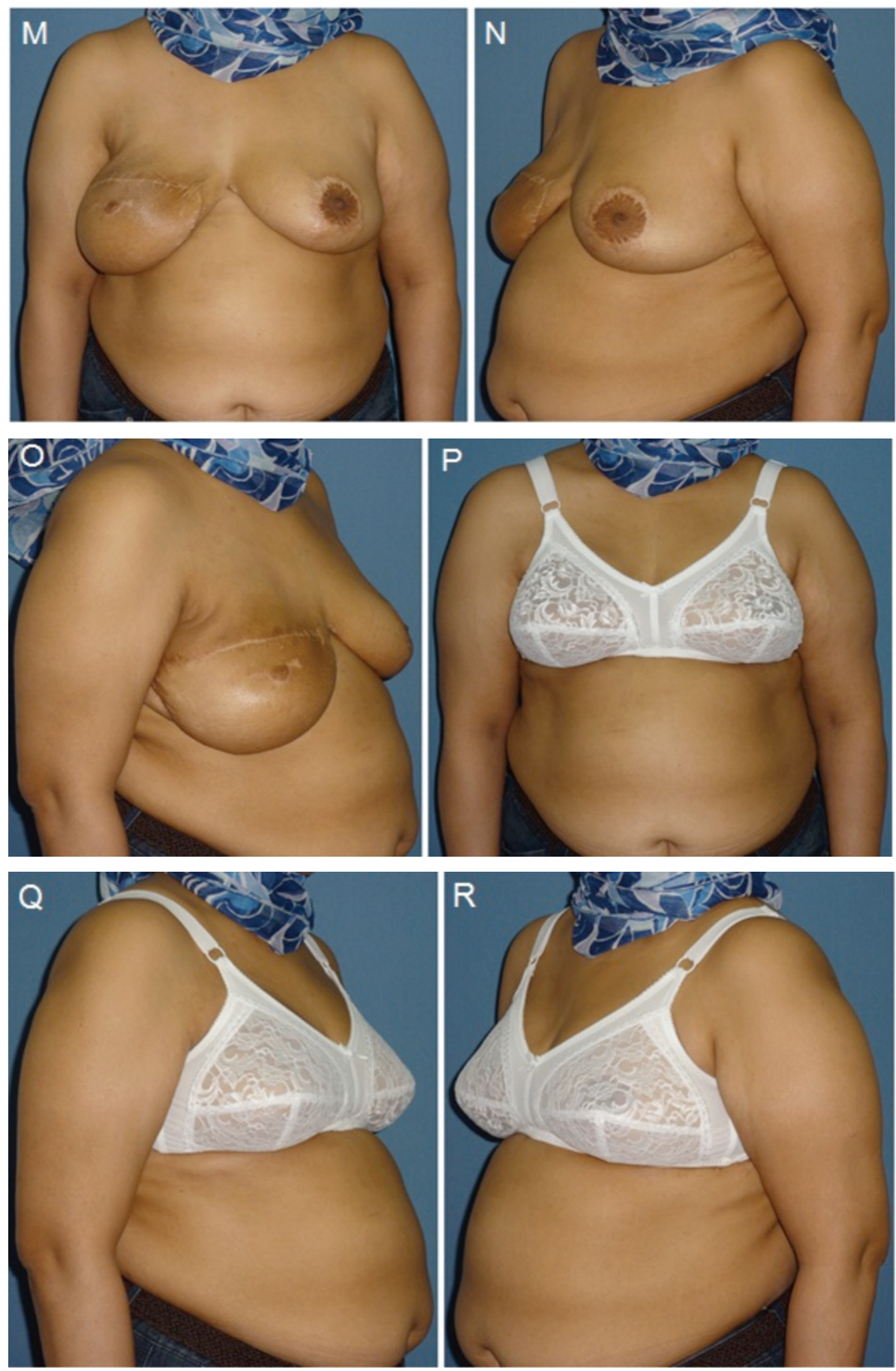

Figure (8): Pre-, intra- and post-operative views of another case with completely survived IMAP flap for postmastectomy reconstruction; $(A, B \& C$ ) Front and oblique pre-operative views. (D) Pre-operative marking of the donor breast. (E\& F) Surgical delay of the lateral flap part, being elevated off the underlying muscle to be re-sutured in place for 2 weeks prior to transposition. $(G)$ Intraoperative view showing the exposed fat at the flap base and the superficial demarcation done to the donor breast markings 2 weeks earlier with temporarily distorted NAC position. (H) A bolster tie-over dressing on a skin graft covering the aforementioned raw area. (I) Pre-division view with graft loss on the flap base. (J)

Immediate intraoperative view after flap pedicle division and completing the left donor breast reduction. Note the intact skin over the sternum. ( $K \& L$ ) Post-operative views 12 weeks after flap division showing a step-off deformity (red circle) at the upper border of the reconstructed breast. Arrow flap for nipple reconstruction was done at the time of deformity correction. ( $M$, $N \& O)$ Post-operative views 12 months after nipple-reconstruction and deformity correction by fat grafting (16 months after flap division). (P) Front view with the bra demonstrating the good symmetry after an overall period of 16 months. $(Q \& R)$ Post-operative oblique views with the bra. 

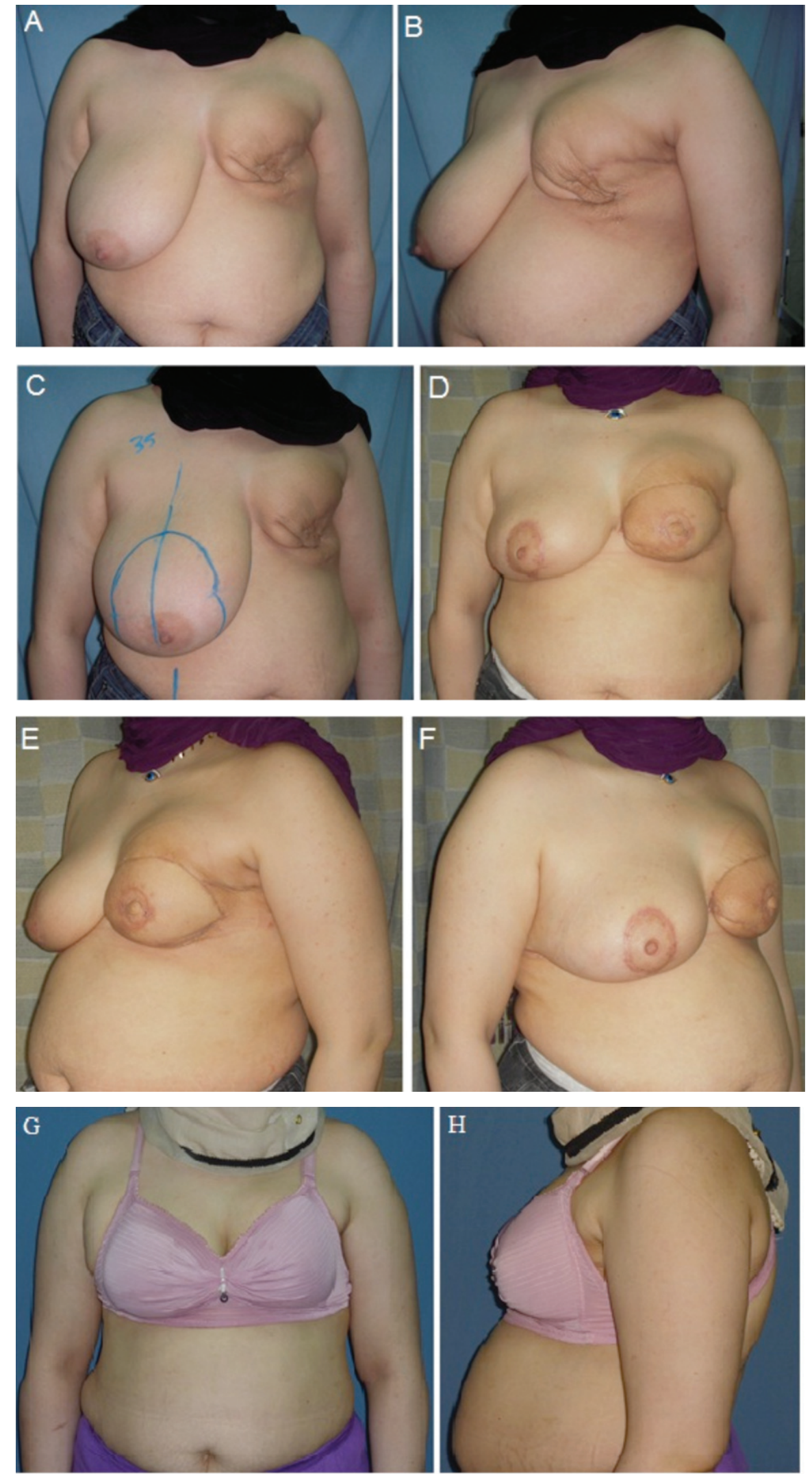

Figure (9): Pre- and post-operative views of the only case with previous left-side submuscular implant reconstruction; ( $A \& B$ ) Pre-operative front and oblique views. Note the radiationaffected skin around the implant. (C) Pre-operative marking of the donor breast. (D-F) Post-operative front and oblique views, 12 weeks after complete reconstruction with IMAP flap, arrow flap for nipple (done 3 weeks earlier), and split-thickness skin graft for areolar reconstruction. Despite the asymmetry observed, the patient expressed her content with the result. (G\&H) Post-operative views with bra after 8 months. 
Table 1 Paients ages, donor breast side and measurements, screened IMAP diameters and remoteness from sternal border, and survived flap dimensions

\begin{tabular}{|c|c|c|c|c|c|c|c|c|}
\hline \multirow[b]{2}{*}{ Case } & \multirow[b]{2}{*}{$\begin{array}{c}\text { Age } \\
\text { [year) }\end{array}$} & \multicolumn{2}{|c|}{ Intact (d on or) breast } & \multicolumn{2}{|c|}{$4^{\text {th }}$ IMAP } & \multicolumn{2}{|c|}{$5^{\text {th }}$ IMAP } & \multirow{2}{*}{$\begin{array}{l}\text { Survived } \\
\text { flap } \\
\text { surface } \\
\text { area }\left(\mathrm{cm}^{2} \text { ) }\right. \\
\text { [Length } \\
\text { "cm" X } \\
\text { Width } \\
\text { "cm"] }\end{array}$} \\
\hline & & Side & $\begin{array}{c}\text { Suprasternal } \\
\text { to NAC } \\
\text { distance }(\mathrm{cm})\end{array}$ & $\begin{array}{c}\text { Diameter } \\
\text { (mm) }\end{array}$ & $\begin{array}{l}\text { Distance } \\
\text { from } \\
\text { sternal } \\
\text { bo rder } \\
\text { (cm) }\end{array}$ & $\begin{array}{c}\text { Diameter } \\
\text { (mm) }\end{array}$ & $\begin{array}{l}\text { Distance } \\
\text { from } \\
\text { sternal } \\
\text { bo rder } \\
\text { (cm) }\end{array}$ & \\
\hline $1^{*}$ & 40 & Left & 42 & 1.3 & 2 & 1.3 & 2.5 & $\begin{array}{c}150 \\
{[15 \times 10]}\end{array}$ \\
\hline $2^{\text {nd }}$ & 30 & Right & 33 & 1 & 1.5 & \multicolumn{2}{|c|}{ Undetectable } & $\begin{array}{c}\text { Total flap } \\
\text { loss }\end{array}$ \\
\hline $3^{r d}$ & 45 & Left & 42 & 1.5 & 1 & \multicolumn{2}{|c|}{ Undetectable } & $\begin{array}{c}253 \\
{[23 \times 11]}\end{array}$ \\
\hline $4^{\text {th }}$ & 37 & Left & 35 & 1.2 & 1.3 & 1.5 & 1.5 & $\begin{array}{c}264 \\
{[24 \times 11]}\end{array}$ \\
\hline $5^{\text {th }}$ & 30 & Right & $2 B$ & 1 & 1.5 & 0.8 & 1.5 & $\begin{array}{c}126 \\
{[14 \times 9]}\end{array}$ \\
\hline $6^{\text {th }}$ & 31 & Right & 35 & 1.1 & 0.5 & 0.6 & 0.5 & $\begin{array}{c}264 \\
{[22 \times 12]}\end{array}$ \\
\hline
\end{tabular}

Table 2 Data concerning the mean, the lowest and highest values for the patientsrelated variables

\begin{tabular}{|c|c|c|c|c|c|c|c|}
\hline & $\begin{array}{c}\text { Age } \\
\text { (year) }\end{array}$ & $\begin{array}{l}\text { Suprasternal } \\
\text { to NAC } \\
\text { distance }(\mathrm{cm})\end{array}$ & $\begin{array}{l}4^{\text {th }} \text { IMAP } \\
\text { diameter } \\
\text { (m m] }\end{array}$ & $\begin{array}{l}4^{\text {th }} \text { IMAP } \\
\text { distance } \\
\text { from } \\
\text { sternal } \\
\text { border } \\
\text { (cm) }\end{array}$ & $\begin{array}{l}\text { Survived } \\
\text { flap } \\
\text { length } \\
\text { (cm) }\end{array}$ & $\begin{array}{l}\text { Survived } \\
\text { flap } \\
\text { width } \\
\text { (cm) }\end{array}$ & $\begin{array}{c}\text { Survived } \\
\text { flap } \\
\text { surface } \\
\text { area } \\
\left(\mathrm{cm}^{2}\right)\end{array}$ \\
\hline Mean & 35.5 & 35.8 & 1.2 & 1.3 & 19.6 & 10.6 & 211.4 \\
\hline SD & 6.2 & 5.4 & 0.2 & 0.5 & 4.7 & 1.1 & 67.7 \\
\hline Minimum & 30 & $2 B$ & 1 & 0.5 & 14 & 9 & 126 \\
\hline Maximum & 45 & 42 & 1.5 & 2 & 24 & 12 & 264 \\
\hline
\end{tabular}

NAC $=$ Nipple-areola complex, IMAP= Internal mammary artery perforator, $\mathbf{S D}=\mathbf{5 t a n d a r d}$ deviation. 
the distal third of the flap, but she refused any further compensatory flap reconstruction. So, re-advancement of the remaining medial part of the flap was performed and after six weeks the flap pedicle division and donor breast remodeling were implemented. Hence, in this six-patient series, two (33.3\%) experienced partial flap loss whereas only one patient $(16.7 \%)$ had total flap necrosis.

On the other hand, the remaining three cases $(50 \%)$ had completely survived IMAP flap with highly-accepted results by the patients both in and out of clothing Figures(7\&8), even in the case with previously-applied submuscular implant (not performed by us) on the side to be reconstructed Figure(9), in spite of the asymmetry discerned between both sides without clothing Figure(9D-F). The autologous fat grafting performed for one case $(16.7 \%)$ of the successful IMAP flap reconstruction to correct step-off deformity was almost totally absorbed after 1 year Figure(8K-O). Tumor-free results through the oncological follow-up (the longest follow-up period for a case reached 29 months while the shortest was 9 months) for both the reconstructed and donor breasts were obtained for all the five patients who had survived IMAP flap-whether partial or total-till date.

\section{Discussion:}

A dermoglandular perforating branch, called IMAP, is given off by the internal mammary artery in each of the first 5 to 6 intercostal spaces laterodorsal to the lateral border of the sternum. These IMAPs traverse superficially in the subcutaneous tissue in a laterocaudal direction to supply the skin of the medial two-thirds of the anterior chest wall in a sequential order, with an overlap of supplied skin zones between consecutive perforators. In females, the 3rd and 4th space perforators tend to be large as they contribute to the arterial supply of the breast. $9,11,20,27$

As far as concerns the $4^{\text {th }}$ and 5th IMAPs, the 4th one has been proven to contribute to the blood supply of the areola, in addition to its typically-supplied skin zone inferior to the areola cranially down to the inframammary fold. The skin of the proximal abdominal wall caudally up to the inframammary fold was evidenced to be nourished by the 5th perforator. $9,11,20,22$

Being proved to be a highly valuable and practical tool providing the necessary information on the vascular anatomy (location, caliber and flow patterns of the perforators) of the flaps, ${ }^{24-26}$ we used the color Duplex preoperatively to detect and ascertain the presence of the $4^{\text {th }}$ and $5^{\text {th }}$ IMAPs or the $4^{\text {th }}$ one at least.

In 2010, Schmidt and colleagues 11 published a comprehensive, descriptive study demonstrating the reliable anatomy (location and size) of the different IMAPs from the $1^{\text {st }}$ one through the $5^{\text {th }}$, and suggesting the variable clinical applications for each IMAPbased flap. Schmidt's study 11 stated that the $5^{\text {th }}$ perforator vascular territory lies below the inframammary fold, and so it's not included within the tissue flap in question. Likewise, we verified, through a clinical study published earlier 23, that the $4^{\text {th }}$ IMAP alone constitutes a reliable vascular pedicle for supplying the inferior pole breast tissues whenever raised as a flap based medially. Therefore, including the $5^{\text {th }}$ IMAP in the flap pedicle wasn't expected to substantially influence the flap perfusion and, furthermore, it confines the rotational capacity of the flap.

According to literature, Aristide Verneuil 28, a French surgeon, was the first to describe the transfer of breast tissue on a pedicle from one side to reconstruct the breast of the opposite side. Yannilos, ${ }^{13}$ in 1950, described, through a case report, a breast sharing reconstruction method using a composite tubed flap transposed from the intact breast on 5 stages. Yannilos ${ }^{13}$ referred to the vasculature of the tubed flap he performed as coming from the perforating branches of the internal mammary artery without any further description to these vessels. Also, in 1980, Franco $^{3}$ reported one case of post mastectomy reconstruction using skin and subcutaneous tissue only flap (without breast tissue involved) derived from the lower pole of the sound breast and based medially, on the top of inserted subcutaneous implant 
two months earlier-what she so called "the dermal-adipose flap". Her procedure was twostaged with one month interval, and she only stated that the flap used was receiving direct ramifications from the internal mammary artery.

Likewise, in 1981, Millard4 published an article addressing the use of skin and subcutaneous tissue only flap taken from one breast to reconstruct the ablated contralateral one on multiple stages (at least three), but, unlike Franco's, ${ }^{3}$ with later insertion of an implant at the final procedure of flap pedicle division. Millard ${ }^{4}$ described his flap as "an economical flap" and named it "inframammary flap", stating that this flap was generously nourished by branches of the IMA without any further illustration. Millard ${ }^{4}$ mentioned that an initial stage of delay is highly indicated as the flap lateral portion was perfused by the lateral thoracic artery.

Actually, the one who is credited for popularizing the breast sharing technique is the Australian plastic surgeon Donald R. Marshall.5,6 He performed his procedure on two stages with six-week interval. Marshal15,6 raised the inferior breast tissue (planned to be discarded in inverted-T reduction mammoplasty) as a medially pedicled flap, starting the incision on the donor breast at the planned new nipple point. Thereby, he transferred the donor breast nipple with the flap, and raised the flap on a broad medial base from the nipple point to the midline. According to Marshall's technique, 5,6 no attempt was made to place the nipple in its planned final position at the time of flap transferring to better preserve the flap vascularity, and postponed this step to the final stage. He pointed out to this vascularity as being dependent on the perforating vessels of the internal mammary chain without identifying which perforators were in charge.

All the above mentioned trials on the employment of the residual breast in reconstructing the missing contralateral one didn't presented enough description for the IMAPs included in the pedicle of the mammary tissue used, and weren't preceded by any means of radiological examination to the flap pedicle vasculature.

In 2001, Schoeller et al. ${ }^{7}$ analyzed preoperatively, with color Doppler sonography, the intact donor breast for the IMAPs to its inferior part, and they found strong perforators in the $4^{\text {th }}$ and 5 th intercostal spaces, but they didn't mention their diameters, their possible numbers, or their exact location within each space. They called the inferior mammary flap they used as "the contralateral split-breast flap", and stated that they performed the procedure via a single stage with subcutaneous transposition of the flap. They reported this method in a single-case work and mentioned that they experienced loss (due to congestion) of the distal third of the flap.

In 2009, a single-case clinical study conducted by gynecologists ${ }^{15}$ described splitting a female breast, but longitudinally rather than transversely, in order to utilize the medial half for contralateral post mastectomy reconstruction. They conducted preoperative Doppler sonographic depiction of the perforators of the IMA supplying the medial half of the donor breast, but didn't present further details about their number, size or location. To the best of our knowledge, this 2009-work was the last-published article, in the English literature, which clinically addressed the concept of breast sharing for post mastectomy reconstruction.

On reviewing all the above-mentioned breast-sharing works, we found the technique we eventually settled on closely resembles Millard's ${ }^{4}$ in terms of the overall number and technicality of stages. The points of disagreement between both techniques relates to the length of intervals between the stages ( 2 then 6 weeks in ours compared to average 3 weeks between each stage in Millard's ${ }^{4}$ ), the delay technique (done to the lateral third of the flap in ours compared to incising the flap all around in Millard's ${ }^{4}$ ), the use of implant at the final stage (unlike Millard ${ }^{4}$, we don't incorporate implants in our procedure), and lastly the composition of the flap used (we use a full thickness lipoglandulo-cutaneous flap compared to Millard's skin and subcutaneous 
only flap 4 ).

The congestion we experienced to the distal third of the flap in one of the late cases despite performing the delay procedure could be attributed to the flap narrow pedicle in relation to its length, as we tried in this case to narrow the pedicle to obtain a more rotational capacity to the flap.

The only disadvantage relating specifically to this flap is the possible risk of a second primary carcinoma. Nonetheless, over the last 50 years, there is a considerable divergence of opinion concerning the actual risk of second primary contralateral breast cancer. In the recent literature, the risk is evaluated at $4-5 \% .29,30$ In women younger than 45 years of age at first diagnosis, contralateral rates were estimated by Gao et al. 29 to be $6.2 \%$ and $11.3 \%$ at 10 and 20 years, respectively. However, the incidence of contralateral breast cancer has been found to be declined with the widespread use of adjuvant systemic therapy, being less by $50 \%$ with the use of tamoxifen and by $20 \%$ with other cytotoxic drugs. Hence, the second primary breast cancer is now an infrequent event. At present, the only 2 groups of women at an increased risk of contralateral breast cancer are those with BRCA mutations i.e. genetic predisposition, and women with a history of mantle irradiation during childhood and adolescence. ${ }^{31}$ So, we are totally convenient that there is no logical reason why the risk should be any higher in having the residual breast tissue on two sides rather than one in patients in whom no prophylactic mastectomy is indicated.

We suggest the "cross-breast flap" as another illustrative name for our presented IMAP flap for post mastectomy reconstruction, based on a well-known concept in the plastic surgery of transferring tissue flaps between corresponding body parts as from one finger (cross-finger flap). To the best of our knowledge, this name has not ever been mentioned in the English literature to describe such procedure.

\section{Conclusion:}

The breast tissue below the areola that is typically excised in superior pedicle inverted-T reductive mammary surgery can reliably be raised as an axial flap, based on the $4^{\text {th }}$ IMAP, to reconstruct the contralateral missing breast in the properly selected patients with possible closure of the donor site via reduction mammoplasty technique, thus achieving very low donor site morbidity. A preoperative color Duplex scanning to ensure the presence of the 4th IMAP is highly advisable, or even a must in order to avoid flap vascular complications.

We see the perfect indication for this procedure in elderly patients (especially postmenopausal), who have no pathological features to suggest a significant risk of a second primary tumor (e.g. a nonlobular histological cause for mastectomy), have a negative family history, and have a large contralateral breast needing to be reduced.

\section{References:}

1- Holmstrom H: The free abdominoplasty flap and its use in breast reconstruction. Scand J Plast Reconstr Surg 1979; 13(3): 423-427.

2- Hartrampf CR, Scheflan M, Black PW: Breast reconstruction with a transverse abdominal island flap. Plast Reconstr Surg 1982; 69(2): 216-225.

3- Franco T: Axial flap from the inferior pole of the breast. Ann Plast Surg 1980; 5(4): 260-265.

4- Millard DR Jr: Reconstruction mammoplasty using an economical flap from the opposite breast. Ann Plast Surg 1981; 6(5): 374-380.

5- Marshall DR, Anstee EJ, Stapleton MJ: Post mastectomy breast reconstruction using a breast sharing technique. $\mathrm{Br} J$ Plast Surg 1981; 34(4): 426-430.

6- Marshall DR: The contralateral breast flap in reconstruction of the breast and chest wall. Ann Plast Surg 1993; 31(6): 508-513.

7- Schoeller T, Bauer T, Haug M, et al: A new contralateral split-breast flap for breast reconstruction and its salvage after complication: An alternative for select patients. Ann Plast Surg 2001; 47(4): 
442-445.

8- Salmon M: Les artères de la glande mammaire. Ann Anat Pathol 1939; 16: 477-500. Quoted from Cunningham L: The anatomy of the arteries and veins of the breast. J Surg Oncol 1977; 9(1): 71-85.

9- Palmer JH, Taylor GI: The vascular territories of the anterior chest wall. $\mathrm{Br} \mathrm{J}$ Plast Surg 1986; 39(3): 287-299.

10-Awad MA: Blood supply of the female breast. M.D. thesis. Faculty of Medicine, Ain Shams University: Cairo, Egypt, 1987. Print.

11-Schmidt M, Aszmann OC, Beck H, et al: The anatomic basis of the internal mammary artery perforator flap: A cadaver study. J Plast Reconstr Aesthet Surg 2010; 63(2): 191-196.

12-Paes EC, Schellekens PP, Hage JJ, et al: A cadaver study of the vascular territories of dominant and nondominant internal mammary artery perforators. Ann Plast Surg 2011; 67(1): 68-72.

13-Yannilos HG: The use of composite tube pedicle in the reconstruction of breast defect with subsequent cosmetic repair of the donor breast. Plast Reconstr Surg 1950; 6(5): 396-399.

14-Kalender V, Aydm H, Karabulut A-B, et al: Breast reconstruction with the internal mammary artery pedicled fasciocutaneous island flap: Description of a new flap. Plast Reconstr Surg 2000; 106(7): 1494-1498.

15-Dian D, Drinovac V, Mylonas I, et al: Worldwide first successful splitting of the breast for a single-procedure reconstruction after mastectomy with maintaining the sensitivity. Arch Gynecol Obstet 2009; 280(4): 539-542.

16-Schwabegger AH, Piza-Katzer H, Pauzenberger $\mathrm{R}$, et al: The internal mammary artery perforator (IMAP) breast-flap harvested from an asymmetric hyperplastic breast for correction of a mild funnel chest deformity. Aesthetic Plast Surg 2011; 35: 928-932.

17-Rüegg EM, Lantieri L, Marchac A: Dual perforator propeller internal mammary arteryperforator(IMAP)flap forsoft-tissue defect of the contralateral clavicular area J Plast Reconstr Aesthet Surg 2012; doi:10.1016/j.bjps.2012.03.009

18-Yu P, Roblin P, Chevray P: Internal mammary artery perforator (IMAP) flap for tracheostoma reconstruction. Head Neck 2006; 28(8): 723-729.

19-Neligan PC, Gullane PJ, Vesely M, et al: The internal mammary artery perforator flap: New variation on an old theme. Plast Reconstr Surg 2007; 119(3): 891-893.

20-Vesely MJ, Murray DJ, Novak CB, et al: The internal mammary artery perforator flap: An anatomical study and a case report. Ann Plast Surg 2007; 58: 156-161.

21-Shayan R, Syme DY, Grinsell D: The IMAP flap for pharygoesophageal reconstruction following stricture release. J Plast Reconstr Aesthet Surg 2012; 65: 810-813.

22-Nakajima H, Maruyama Y, Koda E: The definition of vascular skin territories with prostaglandin E1: The anterior chest, abdomen and thigh-inguinal region. $\mathrm{Br} J$ Plast Surg 1981; 34(3): 258-263.

23-Abdelmonem K, Elshahat A, AbouGamrah S, et al: Color duplex assessment of 4th and $5^{\text {th }}$ internal mammary artery perforators: The pedicles of the medially based lower pole breast flaps. Eplasty 2012; 12: 4.

24-Chang BW, Luethke R, Berg WA, et al: Two-dimensional color Doppler imaging for precision preoperative mapping and size determination of TRAM flap perforators. Plast Reconstr Surg 1994; 93(1): 197-200.

25-Rand RP, Cramer MM, Strandness DE: Color-flow duplex scanning in the preoperative assessment of TRAM flap perforators: A report of 32 consecutive patients. Plast Reconstr Surg 1994; 93(3): 453-459.

26-Blondeel PN, Beyens G, Verhaeghe R, et al: Doppler flowmetry in the planning of perforator flaps. Br J Plast Surg 1998; 51(3): 202-209.

27-Romanes GJ: Cunningham's manual of practical anatomy. London: Oxford University Press (Publisher); 14th edn.; 
1977; 2: p.13-14.

28-Verneuil AA: Memoires de Chirurgie. Paris: G. Masson; 1887. Quoted from: Rozen W-M, Rajkomar A-K, Anavekar $\mathrm{N}-\mathrm{S}$, et al: Post-mastectomy breast reconstruction: A history in evolution. Clin Breast Cancer 2009; 9: 145-154.

29-Gao X, Fisher SG, Emami B: Risk of second primary cancer in the contralateral breast in women treated for early-stage breast cancer: A population-based study.
Int J Radiat Oncol Bio Phys 2003; 56: 1038-1045.

30-Yadav BS, Sharma SC, Patel FD, et al: Second primary in the contralateral breast after treatment of breast cancer. Radiother Oncol 2008; 86: 171-176.

31-Morrow M: Prophylactic mastectomy of the contralateral breast. Breast 2011; 20(3): 108-110. 